\title{
Design and Implementation of An Improved Digital Video Surveillance System
}

Ganiyu Adedayo Ajenikoko ${ }^{1}$, Olusoji Simeon Olaniyan ${ }^{2}$, Ajiboye Victor Tayo ${ }^{3}$, Olusola Joel Oyedele ${ }^{4}$, Ademola Abiodun Adetiran ${ }^{5}$ Awogbade Oluwatobi Joseph ${ }^{6}$, Patrick Ayobami Olawale ${ }^{7}$, Olaide A. Osinubi ${ }^{8}$

${ }^{1,2,3,4,5,6,7,8}$ Department of Electronic and Electrical Engineering, Ladoke Akintola University of Technology, P.M.B, 4000, Ogbomoso, Nigeria.

Corresponding Email: ajeedollar@gmail.com

\begin{abstract}
Video surveillance systems also known as Close Circuit Television (CCTV) is the systematic investigation or monitoring of the actions or communications of one or more persons in a place. Video surveillance has been a key component in ensuring security at various institutions due to the increase in crime rate ranging from armed robbery, kidnapping and recently bomb blasts around the globe which has necessitated its installation in most establishments.This study therefore described the steps involved in designing an improved video surveillance system. The study discussed the theory of improved video surveillance types, components involved, selection of the best equipment and the details of its virtual design. The system was designed to monitor the footages of a particular surrounding with acamera resolution of 1680 by 1050 and the monitor displayed the desired output from a simulated implementation of the system with the router which served as an internet connection that transmitted the signal over the internet to any device with internet access.An improved video surveillance system has therefore become a foremost global strategic tool for fighting the war against terrorism, preventing crime, protecting cooperate assets and enhancing public safety which has made forensic investigations very easier for the police force.
\end{abstract}

Keywords: Improved Video surveillance system, Communications, Security, Footages, Virtual Design, Close Circuit Television, Internet Access.

DOI: $10.7176 /$ CEIS/11-2-07

Publication date: April $30^{\text {th }} 2020$

\section{INTRODUCTION}

The alarming crime rate in various homes and offices around the globe has necessitated the installation of video surveillance security systems in most establishments. The security threats ranging from armed robbery, kidnapping among others has kept the entire globe on their toes to the extent that no one can be sure of his/her security in the next moment [3]. Video surveillance is the systematic investigation of the actions or communications of one or more persons in a place. Video surveillance security systems monitor the activities that go on in areas of the establishment with the use of surveillance cameras $[1,5]$.

Video surveillance is so useful to different types of organizations or institutions. The government and law enforcement agencies make use of it for intelligence gathering, for prevention of crime and protection of citizens [2]. Educational institution make use of it to check the status rules and regulations put in place for students to obey such as dressing code, the banking organizations which helps in monitoring people making transactions in banks. This surveillance can also be used for personal need in a residential environment to monitor activities $[4,8]$.

Surveillance involves the physical presence and use of human senses, example is the use of night and day watchman, a practice that has been in existence for a long time, but this method is not without its shortcomings because man is not omnipresent, so man cannot be in the same place at all times of the day [7]. Therefore, a security surveillance system is required to help a man in keeping record of people's activities at a particular location for a specific period of time. This is known as electronic surveillance such as Close Circuit Television (CCTV) and they are of different specifications and it depends on the system for which it is to be used $[6,10]$.

Close Circuit Television (CCTV) system is an integral component of the security measuresthat may need to be adopted by an institution. The institution's premises may need to bemonitored on a regular basis to ensure safety. Close Circuit Television involves the use of video cameras to transmit a signal to a specific place on a limited set of monitors. It differs from broadcast television in which signal is not openly transmitted $[5,8]$. Though, almost all video cameras fit this definition. This term is often applied to those used for surveillance in areas which might need monitoring such as banks, casinos, airports, military installations and stores. There are different types of CCTV systems: these include;analogue and digital, wired and wireless and their modes of 
operation vary. The basic components are more or less the same: a camera, a lens, a monitor, and (for wired systems) cables that carry the signal from one place to another [1, 3. 6, 9].

Many systems also use video recorders to record the video footage. The camera picks up the signal from the area being monitored via the lens (which determines how far and how much the camera can see, and which is often bought separately) and can be either wired or wireless.In a wired system, the camera sends the signals through a cable to the monitor. In wireless systems, no cable is needed, and the camera broadcasts the signal straight to the monitor. The monitor can be either a simple television set (without tuning capacity) or a Personal Computer (PC) or laptop [3, 7]. Most wired analogue systems use television monitors, while digital and wireless systems tend to use computers as monitors for which remote viewing is possible, often via the internet. For recording purposes, the monitor is accompanied by a video recorder, a Video Cassette Recorder (VCR) for analogue systems, or a Digital Video Recorder (DVR) or Network Video Recorder (NVR)for digital systems [2, $6,10]$.

In industrial plants, CCTV equipment may be used to observe parts of a process from central room, for example when the environment is not conducive for humans, CCTV system may operate continuously or only as required to monitor a particular event [4]. A CCTV systemhelps in surveillance of areas that require security round the clock, helps observe and controls traffic (human), prevents theft/ shoplifting, robbery and other crimes, helps identify and initiates legal procedures against offenders, and raise alarms on approaching dangers or avoidable circumstances.An appropriate CCTV system must justify its expense and meets adequate security requirements $[1,4,8,9]$.

\section{a. Video Surveillance Systems}

Video surveillance system also known as Closed Circuit Television (CCTV) systemis the use of video cameras to transmit a signal to a specific place on a limited set of monitors. The key components of every CCTV system are: single or multiple cameras, recording device and a monitor. Closed Circuit Television systems are broadly divided into two known types, namely: wired CCTV systems and wireless CCTV systems [1, 10].

Wired CCTV systems connect the camera to the recording device and monitors with the help of standard coaxial cables or Unshielded Twisted Pair (UTP) cables or fibre optic cables.Wired CCTV systems provide the best picture quality with zero interference, its camera can be located hundreds of meters away from the recording or monitoring equipment and all its sensors can be run from a single power supply [9]. This system is a preferred choice when good picture quality and economic considerations gain are needed.However, the system cabling and installation can be a tedious task, requiring helps from experts, the system is fixed to a specific area and the camera cannot be easily moved to another location and wireless cameras are relatively more expensive $[2,7]$.

A Wireless CCTV systemcomprises of a wireless camera connected to the recording device and monitor. This system is a preferred choice in specific locations devoid of easy cabling facilities and for individuals requiring an easy-to-install solution. A wireless CCTV system camera is portable, cost effective andcan be moved to other locations requiring observation. The system is best suited for locations requiring temporary observation or in a temporary location.Its camera can be hidden to detect theft or pilferage and its recording and monitoring device need not be in the same line of sight allowing observation of any place from another remote location [4,9]. However, the system requires a dedicated frequency to transmit signals from the camera to the receiving and recording station.Its frequencies may be subjected to various interruptions by the use of electric motored products, air conditioning, fluorescent lighting or cordless telephones which affects the picture quality.Its camera may not provide the best picture quality as such systems are susceptible to picture distortion and require wireless technology-specific expertise to diagnose and fix break downs in the system [5, 7].

\section{b.Applications.}

In its broadest sense, the purpose of an improved video surveillance system(Close Circuit Television) in any security plan is to provide remote eyes for a security operator in order to create live-action displays from a distance. The video system hasa recording means: a VCR, a DVR, or other storage media to maintain permanent records for training or evidence [7]. The followings are some applications for which video provide an effective solution [9]:

i. When overt visual observation of a scene or activity is required from a remote location.

ii. Covert observation of a scene. It is easier to hide a small camera and lens in a target location than to station a person in the area.

iii. There is little action to watch in the area, as in an intrusion-detection location or a storage room, but significant events must be recorded in the area when they occur. 
iv. Integration of video with alarm sensors and a time-lapse/real-time VCR or DVR provides an extremely powerful solution.

v. Many locations must be observed simultaneously by one person from a central security location.

vi. Tracing a person or a vehicle from an entrance into a facility to a final destination. The security force can then predict where the person or vehicle can be interdicted.

vii. Often a guard or security officer must only review a scene for activity periodically. The use of video eliminates the need for a guard to make rounds to remote locations, which is wasteful of a guard's time.

viii. When the crime has been not been committed, capturing the scene using the video camera and recorder to have a permanent record and a hard copy printout of the activity and event is possible.

\section{c. Hardware Components of aVideo Surveillance System}

Some of the hardware components used in a video surveillance system include [7,9]:

i. Lens: light from the illumination scene reflects off the scene. The lens collects the light from the scene and forms an image of the scene on the light-sensitive camera sensor.

ii. Camera: The camera sensor converts the visible scene formed by the lens into an electrical signal suitable for transmission to the remote monitor.

iii. Transmission Link: The transmission media carries the electric video signal from the camera to the remote monitor. Hard-wired media choice includes: - Coaxial, 2-wire Unshielded TwistedPair (UTP), Fibre-optic cable, Local Area Network (LAN), Wide Area Network(WAN), Internet network. While, wireless choice includes: Radio Frequency (RF), Microwave and optical infrared signals can be analogue or digital.

iv. Monitor: The video monitor or computer screens display (Cathode Ray Tube (CRT), Liquid Crystal Display (LCD) or Plasma) and the camera image by converting the electrical video signal back into a visible image on the monitor screens.

v. Recorder: Camera scene is permanently recorded by a real-time or time lapse VCR onto magnetic tape cassette or by a DVR using a magnetic disk hard drive.

vi. Hard-Copy Printer. The video printer produces a hard copy paper printout of any live or recorded video image, using thermal, inkjet, laser or other printing technology.

vii. Camera Switcher, quad Multiplexers: When CCTV security systems have multiple cameras, an electronic switcher, quad, or multiplexer is used to select different cameras automatically or manually to display the images on a single or multiple monitors, as individual or multiple scenes. The quad can digitally combine four cameras. The multiplexer can digitally combine 4,9,16, and even 32 separate cameras.

viii. Housings fall under the categories of indoor, outdoor and integral camera/housing assemblies such as: Dome housing in which the camera uses a hemispherical clear or tinted plastic with pan-tilt-zoom lens capability. In addition, there is plug and play/housing combinations in whichits surveillance camera are packagedin the camera-lens housing.

ix. Pan/Tilt Mechanism: The camera must view a large area span and tilt mount is used to rotate it horizontally (panning) and to tilt it thus. providing a large angular coverage.

x. Splitter/Combiner: An optical or electronic image combiner or splitter is used to display more than one camera scene on a single monitor.

xi. Annotator: Time and date generator annotates the video scene with chronological information. A camera identifier puts a camera number (or name) on the monitor screen to identify the scene displayed on the camera.

\section{d.Video Surveillance SystemCamera}

The type of camera suggests the quality of pictures received for monitoring or recording, Thevideo surveillance systemcamera along with the lens is an important component of the Close Circuit Television(CCTV) system that helps capture images and convert those to electrical signals which are observed or recorded. Although traditional analog cameras are often used in security systems, the trend today is towards digital cameras in video surveillance because they are better to operate in diverse lighting conditions, produce high-quality images andrequiring less storage space than video tapes [6]. Additionally, digital camera users have 
more control setting options, plus Digital Video Recorders (DVRs) which enable users of video surveillance systems to quickly sort through the recorded images in search of a particular incidence or time period $[5,10]$.

To ensure the camera effectively converts light into a video signal; all cameras require an optical lens to focus the light onto an image sensor located directly behind the lens inside the camera. There is a wide variety of cameras and lens available for CCTV systems which makes the task of choosing a system a little difficult [1]. However, there are some considerations that need to be assessed when choosing a camera for CCTV system [3, 9]:

i. Lighting conditions: Image capture during day and night. The level of light has a direct impact on the quality of images.

ii. Position of camera: Indoor or Outdoor. Outdoor camera may require protective enclosures to resist weather and criminals apart from aluminum housing and sealed cable entry.

iii. Field of View: Wide angle or narrow angle- the distance within which objects are in focus.

iv. Focal Length: The distance between the camera and the objects to be recorded.

v. Sensitivity and resolution: Sensitivity means a camera's response to lighting levels while resolution implies the picture quality or details in image produced by the camera.

vi. Color or monochrome: Certain cameras can also switch between color and monochrome depending on lighting conditions. Monochrome cameras are best suitable for low light conditions.

vii. Price: The price of the camera as compared to the features, benefits and after-sales service

In addition,camera data sheet has a number of specifications such asresolution, sensitivity, and signal to noise ratio, camera voltage, chip type, and operating temperature. Some data sheets are detailed, while others are quite sketchy and cover the bare minimum. However, the resolution and sensitivity in the data sheet are normally used to classify a camera. Resolution is the quality of definition and clarity of a picture. It depends upon the number of pixels (picture elements) in the Charge Coupled Device (CCD) chip. In other words the resolution is directly proportional to the number of pixels in the CCD chip while sensitivity is the smallest amount of difference in quality that will change an instrument's reading.On average, native IP-based cameras are less sensitive under low-light than high-end analogue security cameras, and any architecture using native IP cameras must take this into account, interior applications where a well-lit scene can be guaranteed may not need to consider this, but exterior security applications may need to factor this into the camera selection $[6,10]$.

\section{e. Digital Video Recorder}

A Digital Video Recorder (DVR) is a recorder that records motion video in a digital format to a hard disk. This includes various models such as stand-alone, Personal Computer (PC), television, cable or satellite. Sizes include small, portable, desktop, industrial and commercial. Software enables PC to extract segments of video for playback from the hard disk. Closed Circuit Television (CCTV) has long replaced Video Cassette Recorders (VCRs) with digital recorders. These analogue devices initially stored images on magnetic videotapes for playback $[3,6,9]$.

Digital Video Recorders are superior to VCRs because they offer better images, superior search capability, simultaneous recording, live viewing and playback, remote access, easier integration with security systems [2].The DVR is moving from a recording box to a sophisticated system with video management features for example multiplexing, motion detection, neural network processing (the DVR system automatically looks for a specific object that was detected during playback by analyzing the color shape and size of object, then scours the footage for this object), automatic frame rate adjustment, integration with other data sources apart from cameras (e.g. Point of Sale (POS)), and firmware upgrading capabilities [5, 9].

\section{f. Network Video Recorder}

A Network Video Recorder (NVR) is an Internet Protocol (IP) based device that sits on a network. The basic function of an NVR is the simultaneous recording and remote access of live video streams from an IP camera. Most NVRs feature flexible recording and playback capabilities, an optional intuitive remote control unit, a user-friendly Graphic User interface (GUI), intelligent motion detection, and Pan Till Zoom (PTZ) camera control [1].An NVR is a true digital system that receives digital images/ video streams over the network and records them to a hard disk in a digital format. An NVR does not have a dedicated keyboard and monitor. All viewing and management of NVR takes place remotely over the network via a PC [10].

An NVR is designed to offer optimal performance for up to a set number of IP cameras which makes it less scalable than a PC server platform system [4]. This makes the unit suitable for smaller system configurations where the number of cameras stays within the limits of theNVR design capacity. An advantage is that an NVR is less complex to install in comparison to a PC server platform. New generation NVRs hardware are open platform systems that allow the user to run any IP recording software on the market. They usually support 
Windows or Linux environment. These open platform NVRs allow for flexibility and scalability when deploying an enterprise level camera system [10].

\section{g. Video Motion Detector}

Video Motion Detector (VMD) is a component that produces an alarm signal based on a change in the video scene. The VMD can be built into the camera or be a separate component inserted between the camera and the monitor software in a computer [6]. The VMD electronics can be either analogue or digital, store the video frames, compare subsequent frames to the stored frames, then determine whether the scene has changed [5]. In operation, the VMD decides whether a change is significant and whether to flag it as an alarm in order to alert the guard or some equipment or declare it a false alarm [9].

\section{SYSTEM DESCRIPTION OF AN IMPROVED DIGITAL VIDEO SURVEILLANCE SYSTEM}

In the improved Video Surveillance System, the efficiency and accuracy of the system increases as a result of some modifications done to the design and configuration of the system.

The improved video surveillance system was designed and implemented based on three stages; configuration and installation of the digital camera (material used for the installation, hardware specification, installation procedure), transmission of video, monitoring and recording stage. The Digital based camera was configuredand transmittedusing Digital Video Recorder (DVR) to suit purpose for which it was designed.

\section{a. Materials Used for Installation}

The materials used for the Improved CCTV installation comprises of the following:

i. DVR: This was designed to record video streams from the cameras continuously as long as there is a storage device.

ii. Liquid Crystal: This is the visual output unit (monitor) of the surveillance system.It is available to display live surveillance system images.

iii. LAN Cables: It is an Ethernet cable used to connect the surveillance system to the local area networks such as PCs, routers among others.

iv. Digital Cameras: These are devices which do the image capturing while DVR records the captured images.

v. VGA Cable: This is the cable that connects the DVR and the monitor (LCD) together.

vi. Router: This is the device that connects the surveillance system to the internet for remote access and

vii. Power Video Cable: This enables power to be provided to the cameras and other devices. It eliminates the need for power outlet at the camera location.

\section{b. Hardware Specification and Procedures}

The improved video surveillance system was installed and implementedin which four (4) Charge Coupled Device (CCD)Dome indoor cameraswere strategically placed to suit the location as shown in Figure 1. The dome security cameras are popular choice for indoor or outdoor video security. Dome cameras are largely used for monitoring banks and other financial institutions because they are small in size which enables the cameras to be positioned directly so that they are less visible or conspicuous for video surveillance. This camera is very easy to be directed to the location on which it is expected to cover or record. All cameras were connected to the DVR through the power and video cable that was used to power the camera. The hardware specification for the video surveillance system is shown in Table 1.

The camera considered in this study operates on a 12VDC. The power input comprises aplug in, where there is a pair of cable. The pair of cable has red and black color types, symbolizing positive and negative terminals respectively. This cable is fed into the camera from DC power pack which has direct link to the mains. The capture video was transmitting usingthe software based network digital recorder DVR, so that the captured image and videos can be viewed remotely anywhere in the world. The DVR specification is shown in Table 2. 


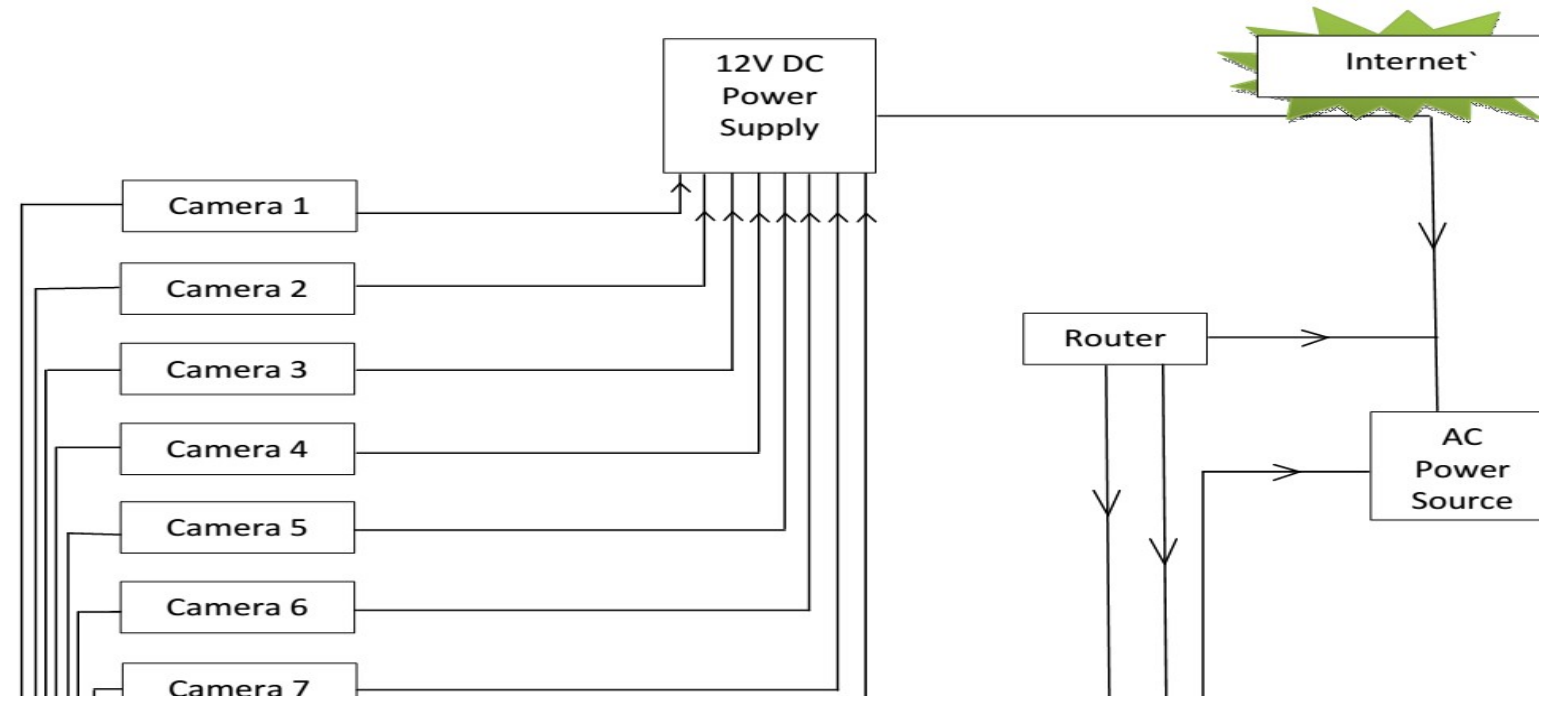

Figure 1: Block diagram of an improved CCTV Surveillance system

Table 1: Hardware Specification

\section{Characteristics $\quad$ Specification}

\begin{tabular}{cc}
\hline Power requirement & $12 \mathrm{Vdc}$ \\
Image sensor & $1 / 4$ LATA color CCD \\
TV line & 700 \\
IR led & 24 \\
Signal & PAL \\
Lens & $6 \mathrm{~mm}$ \\
Weather Proof & Yes \\
IR distance & $700 \mathrm{~m}$ \\
\hline
\end{tabular}


Table 2: DVR recording system specification

\begin{tabular}{ll}
\hline \multicolumn{1}{c}{ Characteristics } & \multicolumn{1}{c}{ Property } \\
\hline Power source & 12VDC \\
Power consumption quality & $80 \mathrm{~W}$ \\
Compression quality & Standard H.264 compression \\
Ports & Simultaneous multi-output, VGA, CVBS and \\
& HDMI (optical) \\
Channels & Eight (8) \\
Internal storage & $500 \mathrm{~GB}$ SATA HDO \\
Connectors & Video power output, high speed serial interface, \\
& $480 \mathrm{Mbps,} \mathrm{Full/half} \mathrm{duplex} \mathrm{RS-485} \mathrm{(RJ-11),}$ \\
& RS232(9-pin D sub), IObaseTx, Ethernet port \\
& (RJ-45) \\
Alarm & Alarm Schedule \\
\hline
\end{tabular}

\section{c. Installation Procedures.}

The procedures for installation of the improved video surveillance system are given as follows:

Step1: The cameras were connected to the power and video cables with the installation pack.

Step 2: Laying of the cable to the above selected position for the cameras to be mounted.

Step3: Mounting of the cameras at the earlier selected positions.

Step 4: Setting up of the power supply to power the DVR, cameras and monitors.

Step 5: Setting up of the digital video recorder (DVR)

Step 5: The camera was positioned to have a wider range of view.

Step 6: Checking from monitor for the expected view and recording of the live footage of the camera.

However, installation of digital surveillance system cannot be done successfully without the use of the storage facilities which actually put down what has been done within a stipulated period of time. Video takes up a staggering amount of space in its original form. As a result of this challenge, video must be compressed, minimized, recorded, stored and transmitted as digital images using High Definition Television (HDTV) which has a recording capacity up to 60 hours.

\section{IMPLEMENTATION OF THE IMPROVED SURVEILLANCE SYSTEM.}

After completing installation of the Digital Video Recorder (DVR) system, a single coaxial cable (Power and Video cables) connected the cameras to the DVR and power supply. The signal was sent to DVR where it was recorded on a hard disk. The DVR was capable of transmitting the video signal line on a monitor connected to the DVR and the router was served as an internet connection that transmitted the signal over the internet to any device with internet access which may be phones, laptops for remote viewing. The improved CCTV camera used has a sensor and a lens. The sensor senses the movement of an intruder while the lens captures the image of the intruder. The resolution of the camera used was $1680 \times 1050$. This is 1680 pixels across the screen (horizontal resolution) and 1050 up and down (vertical resolution).

In accordance with the law of reflection and physics, parallel beam hit the surface of the camera and reflects to converge at the focus where it is collected by the camera and converted into electrical signal that flows through the downlink systems.

However, some of the problems encountered during the implementationinclude:

i. Mounting of the cameras was an issue due to the hardness of the surface on which it was mounted.

ii. Getting the materials was difficult because some of them were not available in our locality

iii. Fading problem as there was constant poor picture quality. 
These problems could be due to error in positioning the CCTV components i.e. the cameras or obstruction in the site location.

\section{CONCLUSION}

This study has analyzed and described the steps involved in designingan improved video surveillance system also known as Improved Close Circuit Television(CCTV)system. Improved Video surveillance systems have extensivelycontributed to situation control by transforming video surveillance from a data acquisition tool to information and intelligence acquisition systems. This system has become a foremost global strategic tool for fighting the war against terrorism, preventing crime, protecting corporate assets and enhancing public safety. Since video surveillance systems serve the needs of military, government, utilities, corporate, medical retail, hospitality, transportation and education, no individual can claim not to be affected by this trend.

This study has broadened our knowledge in the field of CCTV communication. The system prevents forbidden person from entering a secured zone or leaving the suspicious luggage in the office. Although the work involved in the realization of the study was very rigorous, but excellent results were obtained during the testing operation. Therefore, the system will increase security of employees to a larger extent. In addition, the improved video surveillance system has assisted the world to know and identify the intruders that committed a particular crime at a specified time which has made forensic investigations very easier for the security agents.

\section{REFERENCES}

[1] Aleksandra, K. (2011): "Mobile and wireless access in video surveillance system”, International Journal of Digital Information and Wireless Communications (IJDIWC), Vol.1(1), Pp: 267-272.

[2] Jayant, K. (2007): "Understanding CCTV series, camera specifications", www.stamweb.com, Pp: 1-10.

[3] Mwongeera, M. (2009): "Video Surveillance System Design", http://en.wikipedia.org/wiki/Network Video_Recorder, Pp: 1-8.

[4] Neil, C. (2004): "Security: A guide to se3curity systems design and equipment selection and installation", $2^{\text {nd }}$ Edition (revised), Published by: Butterworth-Heinemann, Pp: 1-6.

[5] Ojonemi, P.S., Enejob, A. T. and Olatunmibi, O.A. (2013): "Examination malpractice: challenge to human resource development in Nigeria. International Journal of Capacity Building in Education and Management”, Vol. 2(1), Pp: 91-101.

[6] Ovseník, L., Kolesárová, A. K. and Turán, J. (2010): "Video surveillance systems", Acta Electrotechnica et Informatica, Vol. 10(4), Pp: 46-53.

[7] PSA. (2006): "Requirements for CCTV Installation", The Private Security Authority, Pp: 1-20.

[8] Robb, G.C. (2001): "Police use of CCTV surveillance: constitutional implication and proposed regulation “, University of Michigan Journal of Law RefSorm, Pp. 571-572.

[9] Slawomir, G., Frederic, D., Andreas, K. and Wolfram, B. (2015): "Mapping indoor environments based on human activity", IEEE International Conference on Robotics and Automation, Pp: 34- 36.

[10] Yogameena, B. (2011): "Video Surveillance System-Basics, Essential Guide to Video", Processing by Al Bovik, Academic Press, Pp: 1-20. 